\title{
Multiple-charge beam dynamics in an ion linac
}

\author{
P. N . Ostroumov and K. W. Shepard \\ Physics Division, Argonne National Laboratory, 9700 S. Cass Avenue, Argonne, Illinois 60439
}

(Received 18 November 1999; published 22 March 2000)

\begin{abstract}
An advanced facility for the production of nuclei far from stability could be based on a high-power driver accelerator providing ion beams over the full mass range from protons to uranium. A beam power of several hundred kilowatts is highly desirable for this application. At present, however, the beam power available for the heavier ions would be limited by ion source capabilities. A simple and cost-effective method to enhance the available beam current would be to accelerate multiple charge states through a superconducting ion linac. This paper presents results of numerical simulation of multiple charge state beams through a $1.3 \mathrm{GeV}$ ion linac, the design of which is based on current state-of-the-art superconducting elements. The dynamics of multiple charge state beams are detailed, including the effects of possible errors in rf field parameters and misalignments of transverse focusing elements. The results indicate that operation with multiple charge state beams is not only feasible but straightforward and can increase the beam current by a factor of 3 or more.
\end{abstract}

PACS numbers: 29.17.+w, 29.27. $-\mathrm{a}, 41.75 .-\mathrm{i}$

\section{INTRODUCTION}

Over the past several years, a concept for a rare-isotope facility based on a multibeam ion driver accelerator has been developed [1]. As currently envisioned, the driver would be a $1.3 \mathrm{GeV}$ linac which would accelerate the full mass range of ions and would deliver, for example, several hundred kilowatts of uranium beam at an energy of $400 \mathrm{MeV}$ per nucleon [2,3]. Such a driver can produce large quantities of short-lived exotic isotopes through a variety of nuclear reaction mechanisms, including fragmentation of heavy beams on light targets, in-flight fission of uranium beams, and spallation of heavy targets with light-ion beams [1].

The driver would consist of an electron-cyclotronresonance (ECR) ion source and a short, normally conducting buncher-injector section which would feed beams of virtually any ion into the major portion of the accelerator: an array of more than 400 superconducting (SC) cavities of six different types, ranging in frequency from 58 to $700 \mathrm{MHz}$ [3].

A SC linac is the technology of choice for several reasons. The ability to operate economically in a cw mode minimizes heating problems in the production targets and also makes the best use of existing ion-source technology and performance. The independent phasing of a SC cavity array allows the velocity profile to be varied, permitting higher energies for the lighter ions, for example, $730 \mathrm{MeV}$ for protons. Also, the lower peak current in cw operation reduces space charge effects. This keeps both longitudinal and transverse beam emittance small, and also reduces beam halo. Finally, the short, high-gradient SC cavities provide a linac configuration with very strong transverse and longitudinal focusing, so that the linac acceptance is much larger than the emittance of the ion source and injector.
This latter feature permits a novel method of overcoming the limits of present ion source performance, which, for the heaviest ions, restricts the available beam power to less than the design goal of $400 \mathrm{~kW}$. As we will show, the output beam current can be increased substantially by accelerating more than one charge state through that portion of the linac following the first stripper. An additional benefit of accelerating multiple charge states is the reduction in dumped beam at the stripping points, which appreciably reduces the shielding requirements.

The purpose of this paper is to present a detailed analysis of the dynamics of multiple charge state beams in a SC heavy ion linac suitable as a driver for a rare isotope facility.

\section{DRIVER LINAC OVERVIEW}

A preliminary design and beam dynamics study have been performed for the linac structure shown schematically in Fig. 1. The "prestripper" section of the driver linac consists of an ECR ion source followed by mass and charge selection, an initial linac section consisting of room temperature radio frequency quadrupole (RFQ) and interdigital H-type (IH) structures (up to $\sim 1.3 \mathrm{MeV} / \mathrm{u}$ ), and a section of linac using low-beta SC cavities [4] up to the first stripper at $\sim 1.2 \mathrm{MeV} / \mathrm{u}$. The rest of the linac is based on intermediate-beta SC accelerating structures $[5,6]$. The heaviest ions, which are not fully stripped at the first stripper, will be stripped a second time at $\sim 85 \mathrm{MeV} / \mathrm{u}$. The charge state distribution has been calculated according to Ref. [7]. This distribution is centered at the charge state $q_{0}=75$ for uranium beam. The beam fraction for charge state 75 is $20 \%$; five charges encompass $80 \%$ of the incident beam. As a second stripper, carbon film mounted on the rotating wheel is considered. 


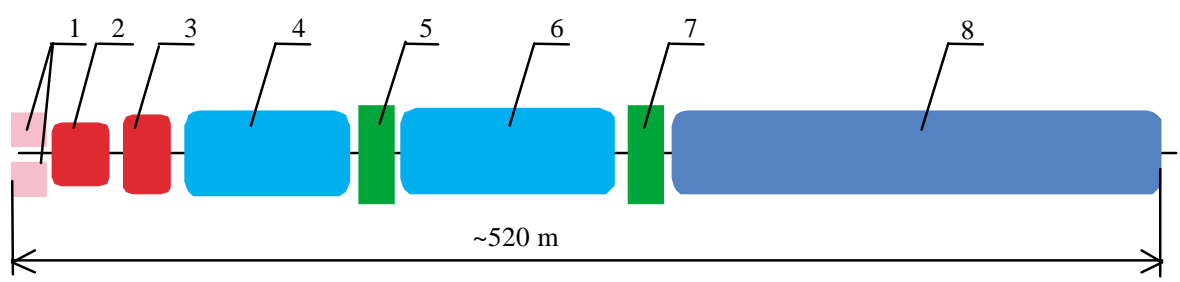

FIG. 1. (Color) General design of the linac. 1: ECR ion sources; 2: $58.3 \mathrm{MHz}$ RFQ; 3: $58.3 \mathrm{MHz} \mathrm{IH}$ accelerating structure; 4: superconducting radio frequency structures operating at 58.3 and $116.7 \mathrm{MHz}$; 5: first stripper $(12.3 \mathrm{MeV} / \mathrm{u})$; 6: SC structures operating at 175 and $350 \mathrm{MHz}$; : second stripper $(85.5 \mathrm{MeV} / \mathrm{u})$; 8: SC structures operating at $700 \mathrm{MHz}$.

After the second stripper, $99 \%$ of the beam is in four charge states neighboring $q_{0}=90$, all of which can be accelerated to the end of the linac.

The resonator configuration for the poststripper linac (downstream of the first stripper) is shown in Table I. Effective accelerating gradients taking into account the cryostat filling are shown in Fig. 2. Transverse beam focusing over all of the driver linac is provided by SC solenoids. The length of the focusing period depends on the resonator type. The parameters of the focusing lattice are listed in Table II. The focusing fields have been optimized for uranium beam.

The required current for production of $400 \mathrm{~kW}$ uranium beam is $0.5 \mathrm{~mA}$. Using the characteristics of the focusing channel from Table II we can estimate the transverse (betatron) oscillation frequency reduction due to the space charge forces [8]. In the case of our application, the ratio of phase advance of the betatron oscillations in one period with space charge $\mu_{t}$ to the phase advance without space charge $\mu_{0}$ is $\mu_{t} / \mu_{0}=0.9987$ at $12 \mathrm{MeV} / \mathrm{u}$, and it becomes even closer to unit at higher energies. The change of the frequency due to the space charge forces is negligible over the entire energy range. As is pointed out in Ref. [8], the space charge fields will play a significant role in the beam dynamics only if $\mu_{t} / \mu_{0} \leq$ $\sqrt{0.5}$. Therefore, in our analysis of multiple charge beam dynamics for this application we can ignore the space charge effects. The reduction of space charge effects obtained by operating $\mathrm{cw}$ mode is one of the main advantages of a superconducting radio frequency (SRF) ion linac for the acceleration of high power beams.

TABLE I. ANL driver linac resonator configuration (poststripper section).

\begin{tabular}{lcccc}
\hline \hline$\beta_{G}$ & Type & $f(\mathrm{MHz})$ & $\mathrm{MV} /$ cavity & $\begin{array}{c}\text { Number of } \\
\text { cavities }\end{array}$ \\
\hline 0.19 & 2 drift tubes & 175.0 & 1.56 & 71 \\
0.38 & 2 spoke & 350.0 & 1.56 & 96 \\
0.488 & 6 cell & 700.0 & 4.33 & 60 \\
0.64 & 6 cell & 700.0 & 7.13 & 96 \\
\hline \hline
\end{tabular}

\section{LONGITUDINAL BEAM DYNAMICS}

\section{A. Analytical approach}

In what follows, we discuss briefly the general case of an ion acceleration in a SC linac and analyze in detail the dynamics of uranium beams, with particular attention to the behavior of multiple charge state beams. We note that for lighter beams the transverse and longitudinal emittances will be smaller and the linac acceptance greater. For this reason, we focus on uranium, with the lowest charge-to-mass ratio, as being the most difficult case.

When a particle with a charge state $q$ and mass number $A$ traverses an accelerating cavity with length $L_{c}$ and electric field $E=E_{g}(z) \cos \omega t$, the energy gain per nucleon, $\Delta W_{n}$, is determined by the expression

$$
\Delta W_{n}=\frac{q}{A} e \int_{0}^{L_{c}} E_{g}(z) \cos \omega t(z) d z .
$$

SRF cavities are designed with a small number of accelerating gaps, configured as either cells or drift tubes. Typically, a cavity is excited in an electric field $\pi$ mode, and the effective cavity length can be defined as $L_{c}=$ $N \beta_{G} \lambda / 2$, where $N$ is the number of accelerating gaps, $\lambda$ is the wavelength of the rf field, and $\beta_{G}$ is the geometric beta of the SRF cavity. The energy gain of a particle

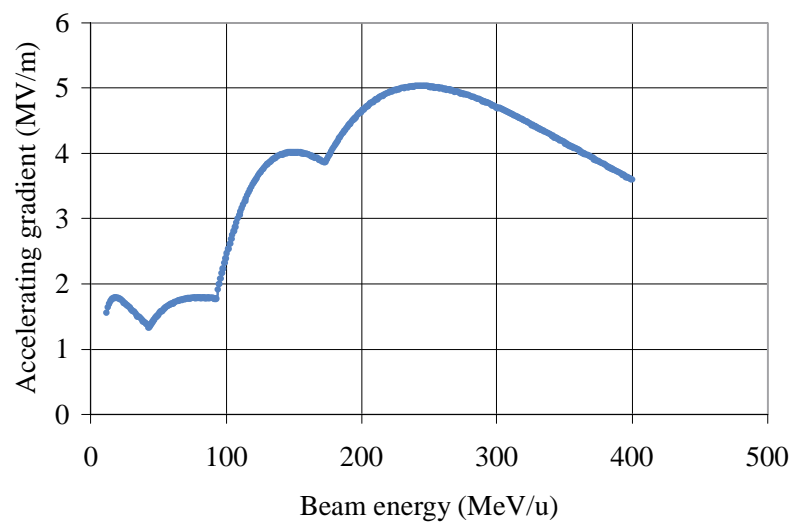

FIG. 2. (Color) Effective accelerating gradient of the driver linac downstream of the first stripper. 
TABLE II. Focusing lattice of the driver linac.

\begin{tabular}{lcccc}
\hline \hline \multicolumn{1}{c}{ Energy range $(\mathrm{MeV} / \mathrm{u})$} & $12.3-42.0$ & $40.3-85.5$ & $85.5-182.3$ & $182.3-400.0$ \\
\hline Operating frequency of accelerating cavities $(\mathrm{MHz})$ & 175 & 350 & 700 & 700 \\
Number of cavities per focusing period & 2 & 4 & 4 & 4 \\
Number of focusing periods & 34 & 24 & 15 & 24 \\
Length of SC solenoid (cm) & 25 & 30 & 60 & 60 \\
Length of the focusing period (m) & 1.66 & 2.8 & 6.5 & 7.5 \\
Focusing field (T) & $5.5-9.3$ & $7.5-10.0$ & $5.4-6.5$ & $6.6-8.7$ \\
Phase advance of betatron oscillation in one period $\left(^{\circ}\right)$ & 60 & 60 & 60 & 60 \\
\hline \hline
\end{tabular}

depends strongly on the input particle velocity $\beta c$. By introducing a transit time factor $T\left(\beta, \beta_{G}\right)$, the energy gain can be rewritten as

$$
\Delta W_{s, n}=\frac{q}{A} e E_{0} T\left(\beta, \beta_{G}\right) L_{c} \cos \varphi_{s},
$$

where $E_{0}=\left(1 / L_{c}\right) \int_{0}^{L_{c}}\left|E_{g}(z)\right| d z$ is the average accelerating field of the cavity and $\varphi_{s}$ is the synchronous phase. The transit time factor (TTF) is a complicated function of both the field distribution and the particle velocity, which may change appreciably during the passage through the multiple gap cavity. For this reason, the TTF is most conveniently calculated numerically.

We define the synchronous phase for a given particle traversing a given field with respect to that rf phase producing maximum energy gain. The synchronous phase, as with the TTF, is generally most conveniently determined numerically. In the case of an odd number of cells and no appreciable velocity change within the cavity, the synchronous phase is the phase of the rf field at the time of arrival at the geometrical center of a symmetric cavity. The field distribution in a three-gap $350 \mathrm{MHz} \mathrm{SC}$ cavity designed for $\beta_{G}=0.38$ is shown in Fig. 3(a). The numerically calculated transit time factor is shown in Fig. 3(b).

The synchronous motion of an ion with charge state $q$ can be considered as motion in an equivalent traveling wave with the amplitude $E_{m}=E_{0} T\left(\beta, \beta_{G}\right)$. We consider the latter as changing adiabatically along the linac for beam energies higher than several $\mathrm{MeV} / \mathrm{u}$.
A heavy ion linac is usually designed for the acceleration of many ion species. In a SC linac the cavities, fed by individual rf power sources, can be independently phased. The phase setting can be changed to vary the velocity profile for synchronous motion along the linac. For a given, fixed, phase setting, the synchronous velocity profile is fixed and $T\left(\beta, \beta_{G}\right)$ is constant. This is similar to the case of a DTL, in which the phase is fixed, and the velocity profile is determined by the DTL geometry. In this case, to accelerate ions with a charge-to-mass ratio $(q / A)_{i}$ different from the design value, the following relation must be satisfied:

$$
\left(\frac{q}{A}\right)_{i} E_{i}=\left(\frac{q}{A}\right)_{0} E_{0} .
$$

That is to say, the phase setting of individual cavities is kept the same as for the design beam, and only the amplitude of the rf field changed in order to fulfill condition (3). In this way, a beam of lower charge-tomass ratio can be accelerated by using higher electric fields. The velocity, and the accelerated beam energy per nucleon, does not depend on the ion species.

In an independently phased cavity array, such as an SRF ion linac, beams of different charge-to-mass ratio can be accommodated by changing either or both the phase and amplitude of the electric field. Allowing both parameters to vary opens the option of varying the velocity profile. This can provide higher energies per nucleon for ions with a higher charge-to-mass ratio. (a)

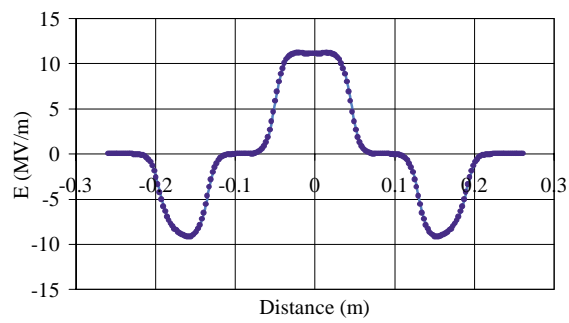

(b)

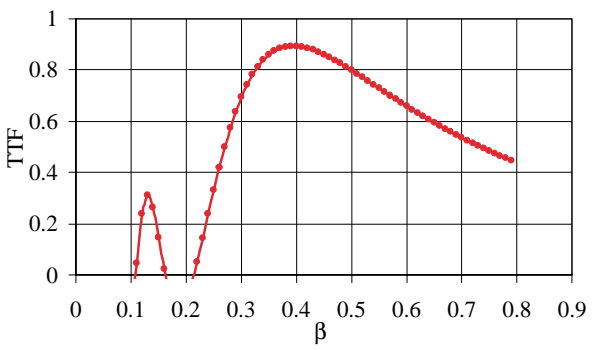

FIG. 3. (Color) Axial electric field distribution (a) and transit time factor (b) of the three-gap $\mathrm{SC}$ spoke cavity, $E_{0} T=5 \mathrm{MV} / \mathrm{m}$, $\beta_{G}=0.38$. 
The driver linac will accelerate uranium ions at charge state $q_{0}=75$ after the first stripper and at $q_{0}=90$ after the second stripper. The simultaneous acceleration of neighboring charge states becomes possible because the high charge-to-mass ratio makes the required phase offsets small. We note that different charge states of equal mass will have the same synchronous velocity profile along the linac if the condition

$$
\left(\frac{q}{A}\right)_{i} \cos \varphi_{s, q}=\frac{q_{0}}{A} \cos \varphi_{s, 0}
$$

is fulfilled. The simultaneous acceleration of ions with different charge state requires an injection of the beam with each charge state $q$ at a synchronous phase which is determined from (4)

$$
\varphi_{s, q}=-\arccos \left(\frac{q_{0}}{q} \cos \varphi_{s, q_{0}}\right) .
$$

Figure 4 shows synchronous phase as a function of charge state calculated for uranium ions at $\varphi_{s, q_{0}}=-30^{\circ}$. This particular example shows that if the linac phase is set for charge state $q_{0}=75$, it can accelerate a wide range of charge states. In fact, 15 charge states of uranium beam could be accepted and accelerated. For $q$ ranging from

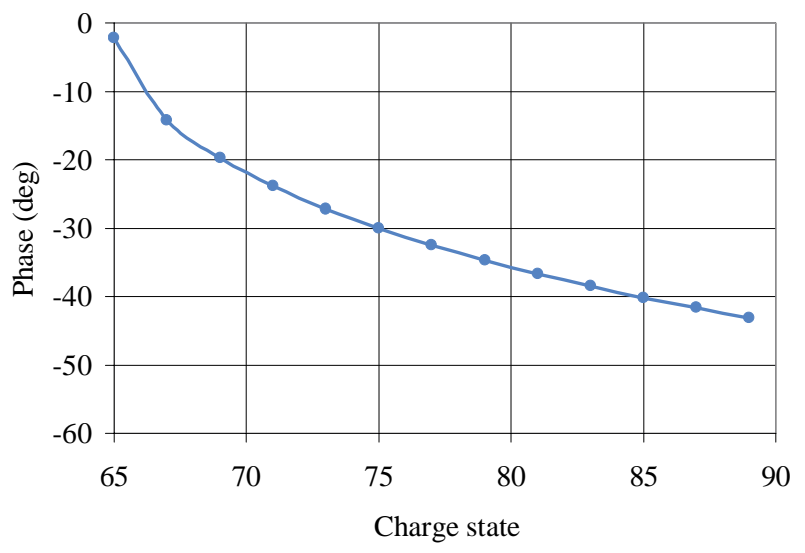

FIG. 4. (Color) Synchronous phase as a function of uranium ion charge state. The designed synchronous phase is $-30^{\circ}$ for $q_{0}=75$.

70 to 85 , even for the worst case charge state of 70 , only a small change in synchronous phase is required, from $30^{\circ}$ to $23^{\circ}$. At this phase, the longitudinal focusing is sufficient to accept the anticipated beam emittance.

The beam stability region in the phase space $[g=$ $\left.\left(p-p_{s}\right) / p_{s}, \varphi\right]$ can be calculated in a conservative approximation and is determined by the expression [9]:

$$
g_{c}(\varphi, q)= \pm \sqrt{\frac{q}{A} \frac{e E_{m} \lambda \gamma}{\pi m_{e} c^{2} \beta}} \sqrt{\left[\varphi-\varphi_{s, q}-\sin \left(\varphi-\varphi_{s, q}\right)\right] \cos \varphi_{s, q}-\left[1+\cos \left(\varphi-\varphi_{s, q}\right)\right] \sin \varphi_{s, q}},
$$

where $m_{e}$ is the atomic unit mass, $\gamma$ is the relativistic factor, and $c$ is the speed of light. The separatrices for charge states $q=73,75$, and 77 are shown in Fig. 5(a).

The phase trajectories of the linear synchrotron oscillations are given by elliptical trajectories

$$
\left(\frac{g}{g_{m}}\right)^{2}+\left(\frac{\Psi}{\Psi_{m}}\right)^{2}=1
$$

(a)

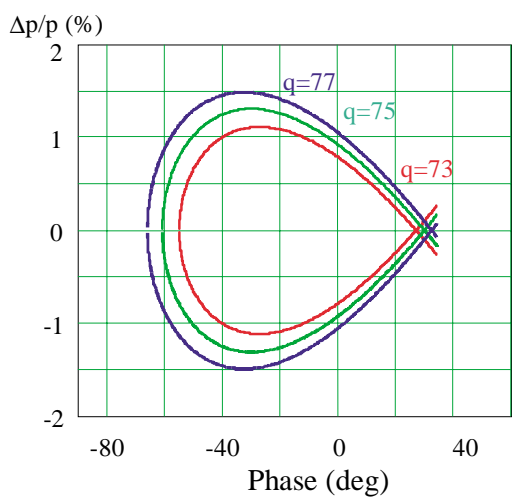

where $\Psi(q)=\varphi-\varphi_{s, q}$ and $\Psi_{m}(q)$ is the amplitude of the phase oscillations. Each particle with different charge state $q$ oscillates around its own synchronous phase with different amplitude as shown in Fig. 5(b). The amplitude of the relative momentum oscillations is

$$
g_{m}(q)=\sqrt{\frac{q}{A} \frac{\gamma_{s} e E_{m} \sin \left|\varphi_{s, q}\right|}{2 \pi \beta_{s} m_{e} c^{2}}} \Psi_{m}(q) .
$$

(b)

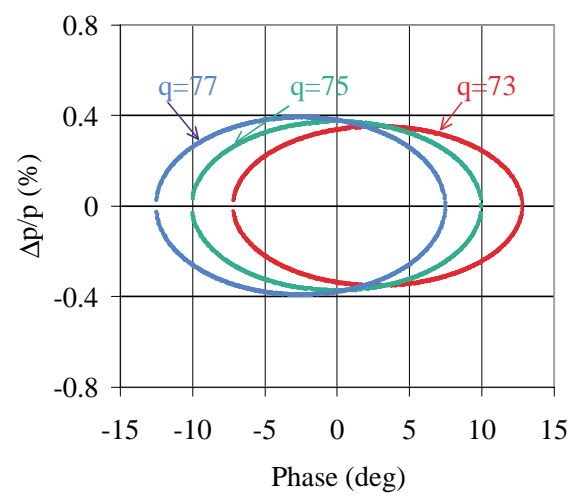

FIG. 5. (Color) Separatrices (a) and small synchrotron oscillation trajectories (b) in the longitudinal phase space for charge states 73,75 , and 77 of uranium beam. 
It would be entirely feasible to eliminate the relative oscillations. If the linac has been tuned for the acceleration of some charge state $q_{0}$, then the particle bunches of different, neighboring charge states could be injected into the linac at different, neighboring rf phases in order for each charge state to be matched precisely to its own phase trajectory. The higher the charge state, the sooner it must arrive at a given point to be matched. One possible method of adjusting the phase of multiple charge states would be a magnetic system, such as a chicane, designed with appropriately varying path lengths for the various charge states.

For the present application, however, such a system is not necessary since the acceleration of a multiple charge state beam is possible even without matching different charge states to the proper synchronous phase. If all charge states are injected at the same time (at the same rf phase), then, as described above, each charge state bunch will perform coherent oscillations with respect to the tuned charge state $q_{0}$. One can view this as an increase in the total (effective) longitudinal emittance of the multiple charge state beam, relative to the (partial) longitudinal emittance of the individual charge state bunches. For the heavy ion SRF linac being considered, the longitudinal emittance is determined by the injector RFQ and can be made as small as $\sim 1 \mathrm{keV} / \mathrm{u}$ nsec for a single charge state beam. For comparison, the linac acceptance, given by the area of the separatrix shown in Fig. 5(a), for $q_{0}=75$ is $77 \mathrm{keV} / \mathrm{u}$ nsec. As will be shown below, this provides ample headroom for the effective emittance growth introduced by the acceleration of multiple charge states.

It should be noted that if no phase matching is done for different charge states, additional emittance growth will occur at frequency transitions in the linac. Heavyion linacs typically have several such transitions to permit efficient operation over the large velocity range required. The additional growth in effective emittance, which can be caused by a frequency transition, is discussed below.

\section{B. Numerical simulations}

To illustrate the feasibility of the concept, we have carried out a Monte Carlo simulation of multiple charge state acceleration from the first stripper through the second stripper and continuing to the end of the linac. The simulation starts with a $12.3 \mathrm{MeV} / \mathrm{u}$ uranium beam equally distributed over five charge states, all at the same rf phase, and with a longitudinal emittance $\sim 1 \mathrm{keV} / \mathrm{u}$ nsec. We consider in detail the behavior of this beam between the two strippers, a section of linac consisting of three-gap SRF cavities operating at 175 and $350 \mathrm{MHz}$. The rf phase throughout this section has been set for acceleration of uranium with charge state $q_{0}=75$ at synchronous phase $\varphi_{s, 75}=-30^{\circ}$. The phase is calculated using values of the electric field numerically generated using realistic cavity geometries, as shown in Fig. 1. The beam tracking simulation was done with a modified version of the LANA code [10]. This code completely simulates beam dynamics in the six-dimensional phase space.

Figure 6 shows the bunch center oscillation of different charge state bunches with respect to the central, $q_{0}=75$, charge state bunch. The phase space plot of all five charge state bunches at $W_{n}=85.5 \mathrm{MeV} / \mathrm{u}$ just before the second stripper, is shown in Fig. 7. As can be seen, the largest increase in emittance occurs at the frequency jump from 175 to $350 \mathrm{MHz}$ cavities. The emittance growth can be minimized if the phase oscillations come to a minimum at the position of the frequency jump. Note that at the second stripper, each of the five charge state bunches is separately mapped into a set of higher charge states which then independently begin processing about their equilibrium points. As a result, after the second stripper, the effective longitudinal emittance of the multiple charge state beam is increased by a factor of $\sim 6$. We note, however, that this longitudinal emittance is still substantially less than the acceptance of the remaining portion of the SC linac. (a)

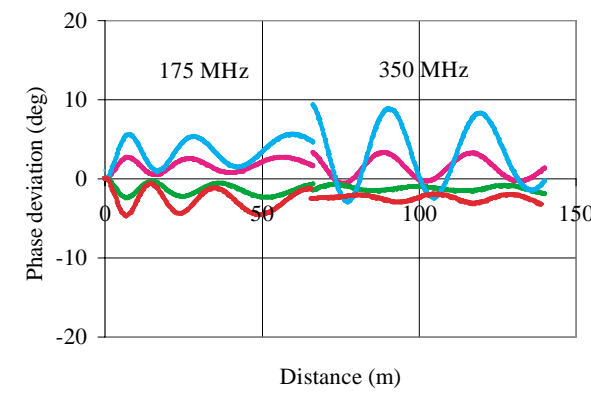

(b)

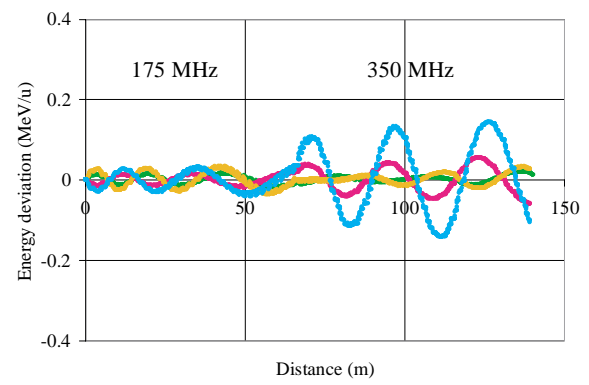

FIG. 6. (Color) Phase (a) and average energy (b) oscillation of multiple charge beam $(q=73,74,76$, and 77$)$ with respect to the charge state $q_{0}=75$. 


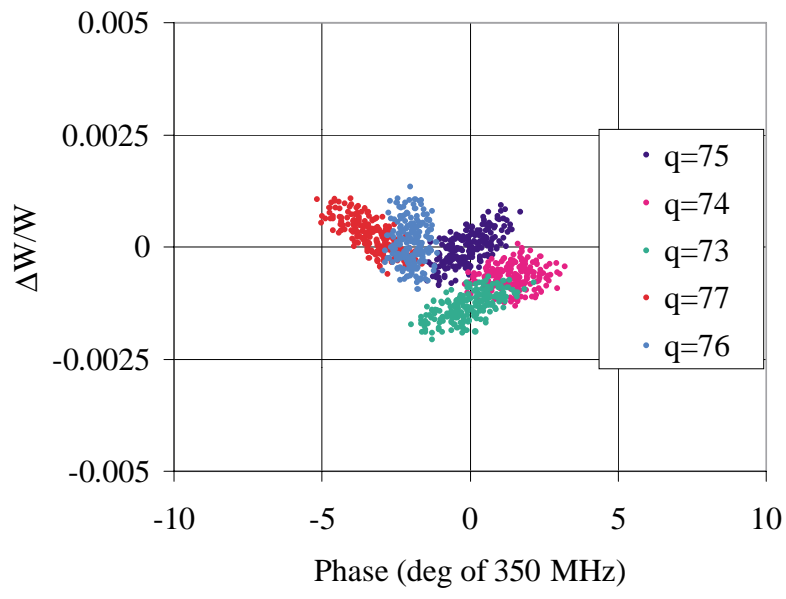

FIG. 7. (Color) Phase space plots of five charge state beam at $85 \mathrm{MeV} / \mathrm{u}$ before passing through the second stripper.

\section{Effects of errors on longitudinal phase space}

The effect of rf field errors on longitudinal beam dynamics in a multicavity linac becomes significant in the present case mainly because of the large number of individual cavities. The rf field errors can be divided into two basic groups. The first is stationary errors, such as a deviation of field level from the designated value and also errors in the designated phase and amplitude settings. Such errors can be minimized by careful time-of-flight measurements, which provide a beam-based experimental determination of the rf field phase and amplitude. A second group of errors is caused by fluctuations, which we assume to be random, in the rf phase and amplitude of the electromagnetic fields in the cavities. This class of error produces growth in the effective longitudinal emittance.

We have performed numerical simulation to estimate the effects of this latter class of error, for both single charge state and multiple charge state beams.

In Fig. 8 we show simulation results for a beam through the 164 cavity section of linac between the first and second strippers. This linac section consists of 68 resonators operating at $175 \mathrm{MHz}$ and 96 cavities at $350 \mathrm{MHz}$. The error effects are included by introducing phase and amplitude errors for each of the cavities, randomly distributed over the indicated range. For such a distribution, the rms error is roughly one-third of the maximum error. The beam was tracked through the linac for a given set of errors so defined, then a new set of random errors generated, and the process repeated. The estimated emittance growth shown in Fig. 8 results from superposing the output beams from 200 such random sets of errors for each value of maximum error. The effective emittance is taken to be that area of phase space required to contain $\sim 90 \%$ of the beam. In these simulations, the area required to contain $100 \%$ of the beam was typically twice as large as for $90 \%$.

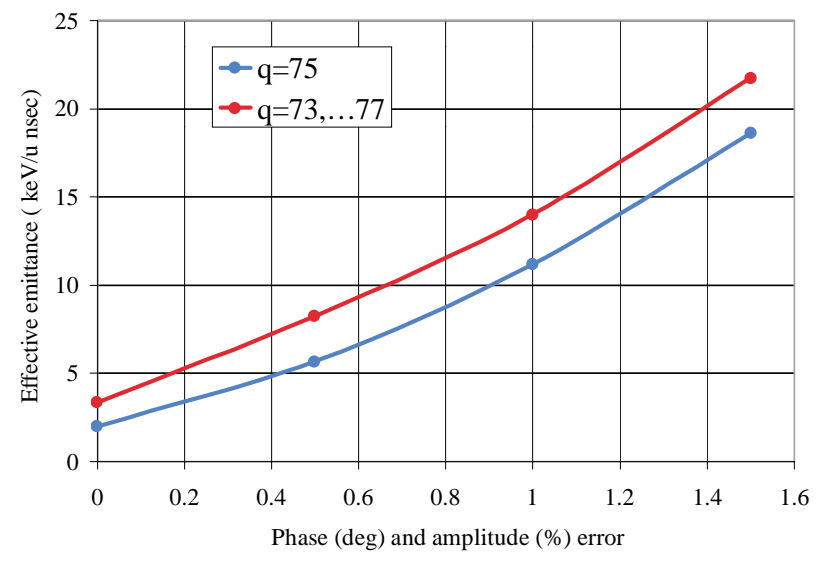

FIG. 8. (Color) Longitudinal emittance as a function of rf phase error for one charge state (blue curve) and five charge state (red curve) beams.

In existing linacs, phase noise in SRF cavities is generally of the order of less than $\pm 0.3^{\circ}$, and amplitude fluctuations are typically less than $\pm 0.3 \%$ (see, for example, [11]). The results shown in Fig. 8 indicate that for such systems the effective emittance growth due to multiplicity of charge states in the beam is much smaller than the growth due to rf noise. Note that even including both these effects, the total increase in longitudinal emittance is still well below the acceptance of the high energy part of the driver linac, $\sim 90 \pi \mathrm{keV} / \mathrm{u}$ nsec.

\section{TRANSVERSE BEAM DYNAMICS}

We now consider the transverse phase space for this same uranium beam through the same section of linac, i.e., between the first and second strippers. The focusing period is defined by a lattice of a SC solenoid following each pair of SRF cavities. The present linac design calls for solenoid focusing elements because SC solenoids are cost effective for this application, but the following analysis is not particularly restricted by this choice.

The matrix of the focusing period is

$$
\begin{aligned}
M & =\left[\begin{array}{ll}
m_{11} & m_{12} \\
m_{21} & m_{22}
\end{array}\right] \\
& =\left[\begin{array}{cc}
\cos \mu_{x}+\alpha_{x} \sin \mu_{x} & \beta_{x} \sin \mu_{x} \\
-\gamma_{x} \sin \mu_{x} & \cos \mu_{x}-\alpha_{x} \sin \mu_{x}
\end{array}\right],
\end{aligned}
$$

where $\alpha_{x}, \beta_{x}$, and $\gamma_{x}$ are Twiss parameters of the matched beam with emittance $\varepsilon_{x}$,

$$
\gamma_{x} x^{2}+2 \alpha_{x} x x^{\prime}+\beta_{x} x^{\prime 2}=\varepsilon_{x},
$$

and $\mu_{x}$ is the phase advance of betatron oscillations over the period [12].

We assume the length of focusing period to be $L_{f}=$ $1660 \mathrm{~mm}$ and the solenoid length to be $L_{s}=250 \mathrm{~mm}$. The magnetic field is varied (from 5.5 to $9.3 \mathrm{~T}$ ) in order 
TABLE III. Twiss parameters of the matched beam at the entrance of solenoid for five charge states.

\begin{tabular}{cccc}
\hline \hline$q$ & $\alpha_{x}$ & $\beta_{x}$ & $\gamma_{x}$ \\
\hline 73 & 0.428 & 1.536 & 0.770 \\
74 & 0.435 & 1.518 & 0.783 \\
75 & 0.441 & 1.500 & 0.783 \\
76 & 0.448 & 1.483 & 0.809 \\
77 & 0.455 & 1.467 & 0.823 \\
\hline \hline
\end{tabular}

to maintain $\mu_{x}=60^{\circ}$, constant along the linac. For solenoidal focusing, the Twiss parameters are identical in the horizontal and vertical planes.

The elements of the matrix $M$ depend on the elements of the lattice and, in our case, can be written as

$$
M=M_{s} M_{d} M_{a} M_{d},
$$

where $M_{s}$ is the matrix of solenoid, $M_{d}$ is the drift space matrix, and $M_{a}$ is the defocusing matrix of the accelerating section. The elements of the focusing period matrix depend, in general, on the charge state of the beam.

For a beam containing multiple charge states, one could, in principle, match each charge state according to its own Twiss parameters. Table III shows the Twiss parameters for five different charge states at $12.3 \mathrm{MeV} / \mathrm{u}$. We can see that the difference in Twiss parameters for five charge states is sufficiently small, that all the charge states can be injected into the linac with the same transverse parameters. If these parameters are chosen to match, for example, charge state $q_{0}=75$, the other charge states will be only slightly mismatched. Using the standard definition [13] for a mismatch factor $K_{m}$, in the worst case, $K_{m}=0.065$ and the effective emittance growth is small.

The transverse beam emittance is determined by the ECR source. Present day ECR sources can produce beam intensities up to $\sim 1 \mathrm{p} \mu \mathrm{A}$ for a single charge state of uranium ions, with an emittance (containing 90\% of particles) equal to $\sim 0.5 \pi \mathrm{mm} \mathrm{mrad}$.

We compare this emittance with the transverse acceptance of the solenoidal focusing channel of the driver linac. For the focusing channel with $\mu_{x}=60^{\circ}$, $\left(\beta_{x}\right)_{\max }=3.17 \mathrm{~mm} / \mathrm{mrad}$. The maximum value of the $\beta_{x}$ function occurs at the center of solenoid, which has a bore radius of $15 \mathrm{~mm}$. This implies a normalized acceptance $A_{n}=11.6 \pi \mathrm{mm} \mathrm{mrad}$.

The acceptance of this section is $\sim 25$ times larger than the beam emittance at entrance. It should be noted that the acceptance of next linac section, the high energy part, is even larger, $\sim 200 \pi \mathrm{mm}$ mrad.

\section{Effects of transverse errors}

The technique we use for error studies for multiple charge beams is nearly identical to the well-established technique for single charge state beams. The particular
TABLE IV. Four types of errors and their tolerable amplitudes.

\begin{tabular}{lc}
\hline \hline \multicolumn{1}{c}{ Error type } & Tolerance \\
\hline 1 Mismatch factor & $20 \%$ \\
2 Transverse displacement of the & \\
focusing lenses & $100 \mu \mathrm{m} \mathrm{each}$ endpoint \\
3 Field and gradient error & $0.3 \%$ \\
4 Rotation of the lenses about the & $30^{\prime}$ \\
longitudinal axis & \\
\hline \hline
\end{tabular}

code for one-charge beam dynamics studies is reported in [14]. The initial distribution in the simulation has been taken as a uniform distribution in four-dimensional phase space. There is no code for the simultaneous simulation of multiple charge beam, therefore we have written our own code. The technique we use for the beam dynamics calculation is essentially that used in the LANA [10], PARMILA, and TRACE [13] codes. The error simulation technique is similar to those described in Ref. [15] but extended for the simultaneous tracking of multiple charge beam. The matrix formalism for the misaligned solenoids has been taken from Ref. [16]. The code uses the Monte Carlo simulation of multiparticle beam dynamics. As mentioned above, we ignore space charge forces. For the error studies the multiparticle simulation is not necessary; strictly speaking, it would be sufficient to study the beam-ellipsoid transformation through the accelerator. We have, however, used the multiparticle code since it is both straightforward and available.

The effects of transverse errors are more severe and less correctable for multiple charge state beams than for single charge state beams for several reasons, as will be discussed below.

Transverse emittance can grow due to several error effects. Table IV shows four main types of errors and typical tolerances. The first type of error, mismatch, is caused by errors in tuning or matching the beam into

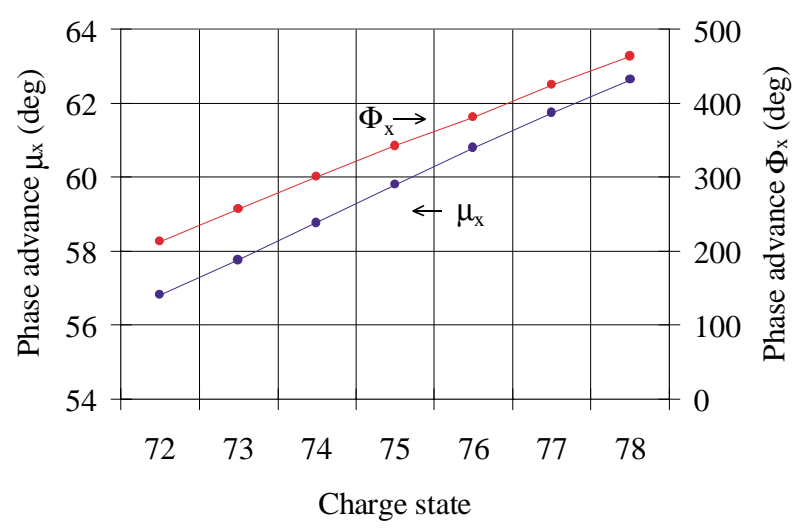

FIG. 9. (Color) Phase advance over the period $\mu_{x}$ and total phase advance $\Phi_{x}\left(\right.$ modulo $\left.360^{\circ}\right)$. 


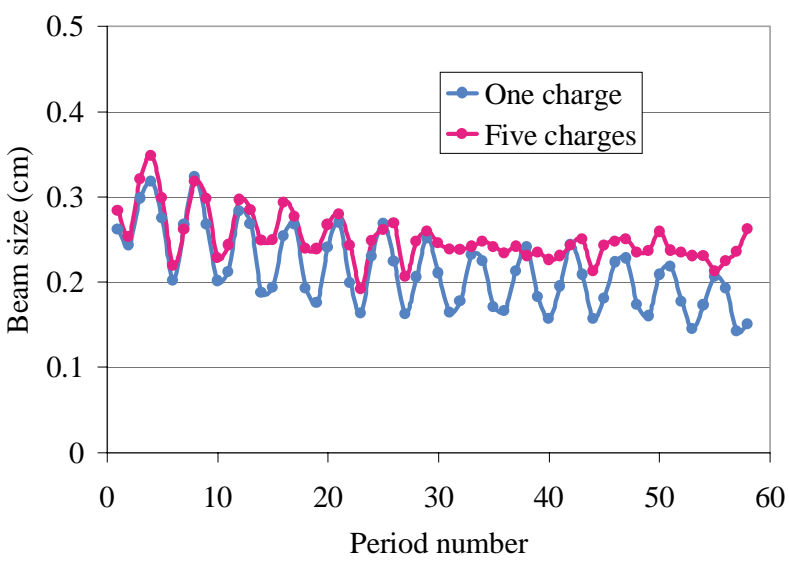

FIG. 10. (Color) Transverse beam envelopes for one charge state beam (blue dots) and five charge state beam (magenta dots). Input beam is mismatched by a factor of 1.4 .

the linac and arises because of errors in measurement of the input beam parameters: we assume a value of $20 \%$, typical of modern linacs [17]. The second type of error can generally be corrected by using steering magnets to offset any measured deviation of the beam centroid.

The fourth class of error is well known to be a major source of emittance growth in a typical ion linac with quadrupole focusing. The driver linac being considered here, however, will employ SC solenoids exclusively as the transverse focusing elements. A benefit of this choice is that the rotational symmetry of solenoids eliminates the effects of rotational errors.

For a single charge state beam, coherent transverse oscillations and rotation in phase space will not increase effective transverse emittance. For this case, the errors discussed above are often of little consequence and can be easily corrected. In the case of a multiple charge state beam, however, the different charge states have different betatron periods. As the beam proceeds along the linac, the transverse oscillations of the various charge states eventually become uncorrelated and the effective total emittance, summed over all charge states, increases.

One aspect of this behavior can be illustrated by considering a mismatched beam through the 58 focusing periods of linac between the two strippers. While the actual linac lattice will be slightly more complex, it is sufficient for us to consider the periodic focusing structure as having constant length. We assume the solenoids to be tuned for a phase advance over one period of $\mu_{x}=60^{\circ}$ for charge state 75 . Figure 9 shows the variation of the transverse phase advance over both a single period and the whole focusing system (58 periods) for the range of charge states being considered.

Although the phase advance per period does not depend strongly on the charge state, over 58 periods the phase differences between different charge states become appreciable. If the input beam is mismatched, the phase space ellipse begins to rotate, at twice the betatron frequency, tracing out a (matched) ellipse of larger area. Figure 10 shows beam envelopes for both a single charge state beam and a five charge state beam. The oscillations of the mismatched beam remain coherent for the single charge state, but not for the multiple charge state case.

The input and output phase space ellipses for mismatched beam are shown in Fig. 11. At the entrance, all five charge states have the same transverse phase space distribution, shown as a blue line in Fig. 11(a). Because the phase ellipse is not matched, it processes, indicated by the green ellipse. The different charge states, however, process at different rates and, after 58 periods, are distributed as shown in Fig. 11(b). At this point, just prior to the second stripper, the multiple charge state beam has expanded to fill the area inside the ellipse delineated by the green curve in Fig. 11.

To summarize, the main difference between one charge state beams and multiple charge state beams is that (a)

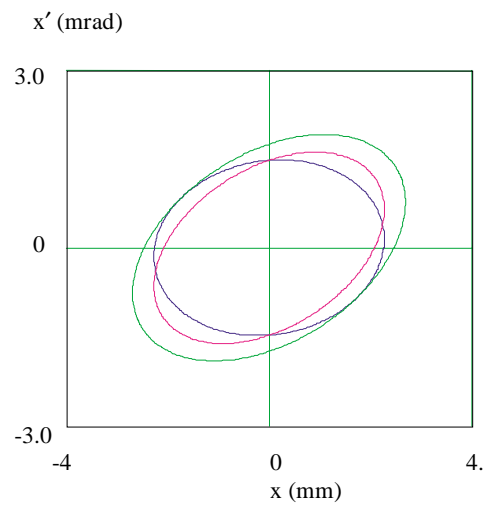

(b)

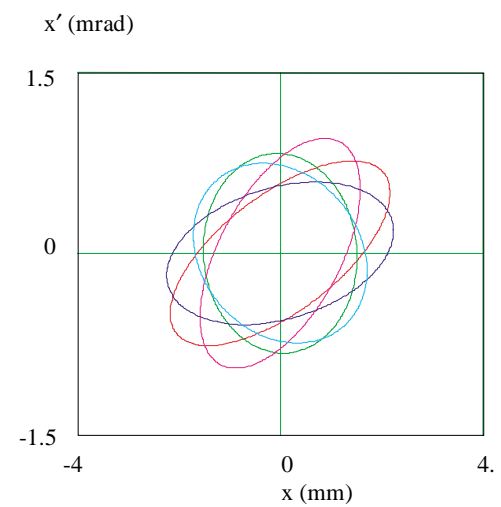

FIG. 11. (Color) Transverse ellipses of mismatched beam (mismatched factor is 1.4) at the entrance (a) and exit (b) of the periodical focusing channel. The left figure shows the matched ellipse (magenta), mismatched ellipse (blue), and effective emittance (green). 
mismatch of a single charge state beam is generally correctable and does not lead to transverse emittance growth; for multiple charge states, correction is more difficult and will generally induce growth in transverse emittance.

Multiple charge state beams are also more severely affected by misalignment errors. Misalignments produce a transverse magnetic field on the linac axis and coherently deflect the beam. For a single charge state beam, misalignment causes lateral displacement of the beam, but no emittance growth so long as the beam remains in the linear region of the focusing elements. With a beam containing multiple charge states, the differing betatron periods, as well as the differing displacements, cause growth in the transverse emittance. As will be shown below, the usual corrective, steering, can only partially correct this situation.

We have performed Monte Carlo simulations of the dynamics of multiple charge state beams in the presence of alignment errors. As in the previous sections, we consider a five charge state uranium beam in that portion of the linac between the first and second strippers. To make the simulation more realistic, we assumed a mismatch factor of 1.2 for the beam out of the first stripper. We introduced alignment errors by displacing separately both ends of each of the 58 focusing solenoids in both $x$ and $y$ by an amount randomly varying over the range $\pm 100 \mu \mathrm{m}$. Then we tracked the multiple charge state beam through this portion of the linac and noted the increase in transverse emittance resulting from the positioning errors. This entire simulation was then repeated 200 times, each time with a different, random set of alignment errors. The red bars in Fig. 12 are a histogram of the results. The emittance growth factor is the ratio of (normalized) transverse emittance of the beam at exit to that at entrance. Note that for some sets of alignment errors the emittance growth factor can be as high as 8.5.

Even for multiple charge state beams, however, emittance growth can be substantially reduced by simple corrective steering procedures. We have modeled this by assuming a measurement of beam centroid position and corrective steering to be performed once every four focusing periods. This interval would correspond to the space between cryomodules in the benchmark linac design.

As will be discussed below, the transverse tune has important effects. We consider the case of a $60^{\circ}$ phase advance per focusing period. For this case, the phase advance between the points at which monitoring and steering is performed is $\Phi_{x}=240^{\circ}$. This rotation in transverse phase space transforms a centroid displacement at one corrective station to a deflection at the succeeding station, which can be directly corrected by simple oneelement steering at that point.

In our simulations, we assumed a centroid measurement error of $0.1 \mathrm{~mm}$, and a steering field error of $0.1 \mathrm{mrad}$.

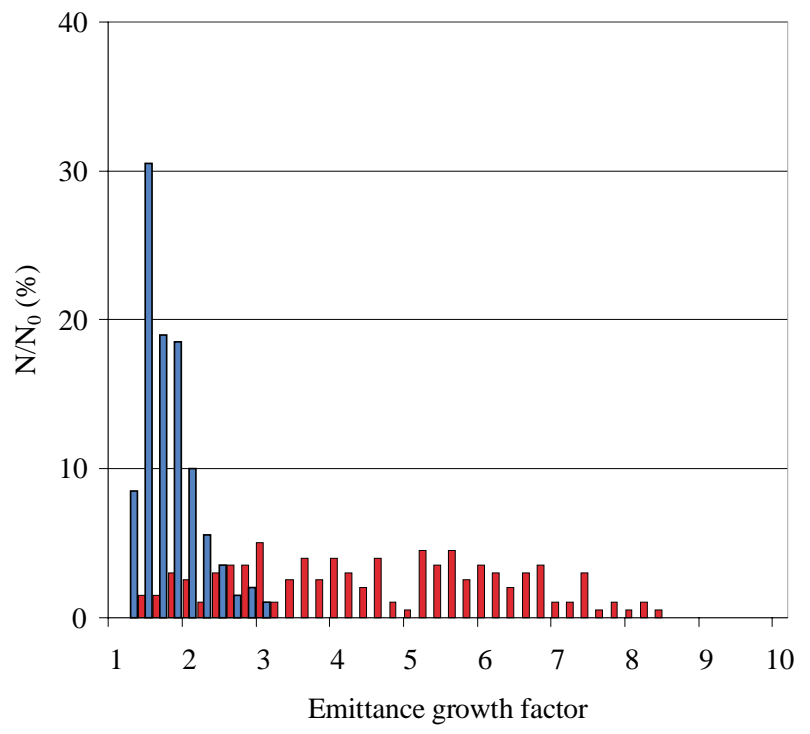

FIG. 12. (Color) Emittance growth in the misaligned focusing channel with $\mu=60^{\circ}$. Vertical axis is a percentage of simulations with the indicated emittance growth, $N_{0}=200$. The blue bars show a case with the beam steering; the red bars show a case without steering.

The beam center was calculated as the center of gravity of a five charge state beam with $q=73, \ldots, 77$. The results are shown by the blue bars in Fig. 12. It can be seen that the emittance growth factor has, in all cases, been reduced to less than three. For the entire set of cases of random errors, the most probable value for the effective emittance growth factor is 1.4.

For different transverse tuning, steering may not be as effective as shown in Fig. 12. We have performed the same simulation for the case of the focusing channel tuned for a phase advance of $\mu_{x}=40^{\circ}$ per period. The beam steering procedure is less effective in this case. Beam emittance growth up to a factor of 8 occurs for some error distributions, even when steering corrections are applied. This example shows that the choice of the focusing structure parameters can be important in order to avoid large emittance growth of multiple charge state beams due to misalignments.

The emittance growth of mismatched multiple charge beam has been studied in the presence of misalignments and beam center steering procedures. The results are shown in Fig. 13, where the emittance growth factor is shown as a function of the entrance mismatch for the case of no alignment errors and for the case of alignment errors corrected by steering. In the case of alignment errors, the emittance growth factor was taken to be that value larger than that produced by $90 \%$ of the 200 cases of random alignment error simulated. Figure 13 shows that mismatching and misalignment will contribute in roughly equal measure to the total emittance growth of multiple charge state beams. 


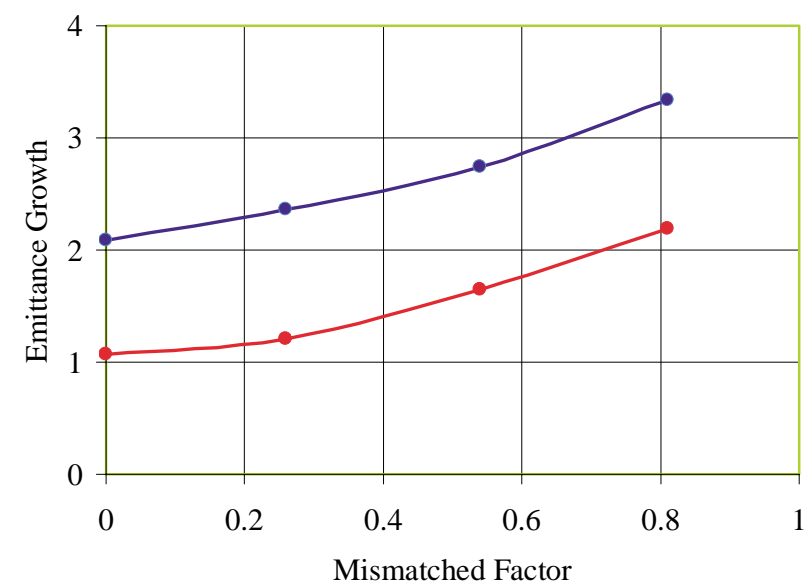

FIG. 13. (Color) Full emittance growth of five charge state beam versus mismatched factor in the focusing channel without errors (red dots) and with errors (blue dots).

\section{CONCLUSIONS}

The large longitudinal and transverse acceptance characteristics of superconducting heavy-ion linacs makes possible the acceleration of multiple charge state beams. We have performed numerical simulation of such beams for a benchmark design driver linac for a rare-isotope production facility. The results indicate that it is quite feasible to accelerate five charge states of uranium after the first stripper and four charge states after the second stripper in this linac.

Such operation could provide $61 \mathrm{~kW}$ of uranium beam using a demonstrated ECR ion source, the AECR-U at the Lawrence Berkeley National Laboratory, which has been shown to produce $0.8 \mathrm{p} \mu \mathrm{A}$ of uranium beam at charge state 30 [18].

A very preliminary analysis indicates that it may be feasible to extend this method to the injector section of the linac, and accelerate two charge states through the $58 \mathrm{MHz}$ RFQ. The RFQ on the lead injector at CERN has accelerated several charge states of Ta ions from a high charge state laser ion source [19]. Details of the beam dynamics in this area need further study, but initial calculations are promising. If such operation proves possible, then a $120 \mathrm{~kW}$ uranium beam can be achieved with present day ion source performance.

\section{ACKNOWLEDGMENTS}

The authors thank J. Staples of LBNL for insightful discussions in the initial stage of this work. We also thank J. Nolen and M. Portillo for discussion of their ongoing study of the optics of magnet systems for multiple charge state ion beams. This work was supported by the U.S. Department of Energy, Nuclear Physics Division, under Contract No. W-31-109-ENG-38.
[1] J. A. Nolen et al., in Heavy Ion Accelerator Technology: Eighth International Conference, edited by K. W. Shepard, AIP Conf. Proc. No. 473 (AIP, College Park, MD, 1999), p. 477; Argonne National Laboratory Report No. ATLAS99-1, 1999.

[2] P. N. Ostroumov, K. W. Shepard, J. A. Nolen, and R. C. Pardo, in ICFA Beam Dynamics Newsletter 20, 60 (1999).

[3] K. W. Shepard et al., in Proceedings of the 9th International Workshop on RF Superconductivity, Santa Fe, New Mexico, 1999 (to be published).

[4] K. W. Shepard, in Proceedings of the 7th International Conference on Heavy Ion Accelerator Technology, Canberra, Australia, 1995 [Nucl. Instrum. Methods Phys. Res., Sect. A 386, 132 (1996)].

[5] B. Rusnak et al., in Proceedings of the XIX International Linac Conference, Chicago, 1998, edited by C. E. Eyberger, R. C. Pardo, and M. M. White (Argonne National Laboratory Report No. ANL-98/28, 1998), p. 986.

[6] K. W. Shepard et al., in Proceedings of the 1999 Particle Accelerator Conference, New York (IEEE, Piscataway, NJ, 1999).

[7] A. Leon, At. Data Nucl. Data Tables 69, 2 (1998).

[8] M. Reiser, Theory and Design of Charged Particle Beams (Wiley, New York, 1994).

[9] I. M. Kapchinsky, Beam Dynamics in Linear Resonance Accelerators (Atomizdat, Moscow, 1966), in Russian.

[10] D. V. Gorelov, P. N. Ostroumov, and R. Laxdal, in Proceedings of the 1997 Particle Accelerator Conference, Vancouver, Canada, edited by M. Comyn, M. K. Craddock, M. Reiser, and J. Thomson (IEEE, Piscataway, NJ, 1998).

[11] S. Simrock, in Proceedings of the 1991 Particle Accelerator Conference, San Francisco (IEEE, New York, 1991), p. 2515.

[12] J. Rossbach and P. Schmuser, in Proceedings of the Fifth General CERN Accelerator School, University of Jyvaskyla, Finland, 1992 (CERN, Geneva, 1994), p. 17.

[13] K. W. Crandall, Los Alamos National Laboratory Report No. LA-11054-MS, 1987.

[14] D. V. Gorelov and P. N. Ostroumov, in Proceedings of the XIX International Linac Conference, Chicago, 1998 (Ref. [5]), p. 654.

[15] A. D. Vlasov, Theory of Linear Accelerators (Israel Program for Scientific Translations, Jerusalem, 1968), translated from Russian.

[16] B. I. Bondarev et al., in Proceedings of the 1972 Proton Linear Accelerator Conference, Los Alamos, New Mexico, edited by K. H. Harper (LANL Report No. LA-5115, UC28, 1972), p. 119.

[17] K.F. Johnson et al., in Proceedings of the 1992 Linac Conference Ottawa, Canada, edited by C. R. Hoffmann (Chalk River Laboratories Report No. AECL-10728, 1992), p. 64.

[18] C. M. Lyneis et al., Rev. Sci. Instrum. 69, 682 (1998).

[19] M. Bourgeois et al., in Proceedings of the 1996 Linac Conference, Geneva, Switzerland, edited by H. Haseroth (CERN, Geneva, 1996), p. 64. 